\title{
Salivary Alpha-Amylase Reactivity under Psycho-Physiological Stress. A Nonverbal Communication Measurement Tool?
}

\author{
Takuji Inagaki $^{1}$, Masa Ieda ${ }^{2}$, Satoko Yamashita ${ }^{2}$, Tsuyoshi Miyaoka ${ }^{2}$, Jun Horiguchi ${ }^{2}$ \\ ${ }^{1}$ Shimane University, Faculty of Education, Department of Psychology and Special Support Education, \\ Nishikawatsu, Matsue, Shimane, Japan \\ ${ }^{2}$ Shimane University, Faculty of Medicine, Department of Psychiatry, Shimane, Japan \\ E-mail: inagaki@edu.shimane-u.ac.jp \\ Received January 25, 2011; revised February 12, 2011; accepted February 17, 2011
}

\begin{abstract}
Previous studies have shown that changes in salivary alpha-amylase (sAA) levels are dependent on psychosocial stress stimulation and reflect the activity of the sympathetic nervous system. sAA measurement can be performed easily and quickly; therefore, it may be useful for evaluating psychosocial or physical stress. The aim of this preliminary study was to examine the use of sAA measurements as objective indicators of psychosocial and/or physical stress levels by examining sAA changes in volunteers subjected to conditions similar to those suffered by children with severe motor and intellectual disabilities and cerebral paralysis. Twelve healthy volunteers were required to not move or speak, as is found in patients suffering from total paralysis, for $30 \mathrm{~min}$. Saliva samples were taken at three points, and sAA activity was measured using a hand-held monitor before the test, immediately after the test, and 10 min after the test. In the present study, a marked increase in sAA activity due to physiological stress and a rapid return to the baseline level were observed. Many subjects felt body pain and mental distress. This measurement method is useful for evaluating stress in children with severe motor and intellectual disabilities, who can not fully express their emotions or communicate with their caregivers.
\end{abstract}

Keywords: Nonverbal Communication, Psychosocial Stress, Physical Stress, Salivary Alpha-Amylase, Severe Motor and Intellectual Disabilities

\section{Introduction}

Salivary alpha-amylase (sAA) has been suggested to reflect stress-related body changes. Psychosocial stress increases the release of salivary alpha-amylase, which reflects the activity of the sympathetic-adrenal-medullary (SAM) system [1-3]. Therefore, it is supposed that sAA measurement is a useful tool for evaluating the SAM system. In addition, previous studies examining the response of sAA levels to SAM system activity showed that increased sAA levels were correlated with increased plasma catecholamine, indicating sympathetic nervous system activation $[4,5]$. To date, numerous studies have shown that changes in sAA levels are dependent on stress stimuli [1,6-10].

As far as we know, few studies have investigated psychosocial or physical stress in children with severe motor and intellectual disabilities such as patients with severe cerebral palsy. Such patients usually can not fully communicate with others or describe their emotions. It is therefore difficult to objectively evaluate their emotional and physical state. sAA measurement can be performed easily and quickly, and therefore, could be used to aid the evaluation of the psychosocial and/or physical stress levels of such patients.

In special support education, it is very important to be able to evaluate and understand the level of psychosocial and/or physical stress (distress) experienced by a child, particularly in children with severe motor and intellectual disabilities. The measurement of sAA is expected to be useful as a nonverbal communication procedure for children with severe motor and intellectual disabilities.

The aim of this preliminary study was to examine the use of sAA measurements as objective indicators of 
psychosocial and physical stress levels by examining sAA changes in volunteers subjected to conditions similar to those suffered by children with severe motor and intellectual disabilities and cerebral paralysis; i.e., the participants were ordered to lie down with their face pointing upward and to not move or speak for thirty minutes.

\section{Methods}

The study subjects were recruited at Shimane University. Twelve healthy student volunteers (mean age: $20.8 \pm 1.8$ years; 7 females; 5 males) were recruited from the Department of Psychology and Special Support Education at the university. All subjects gave their written informed consent before participating in this study after the purpose and procedure had been fully explained to them. All subjects were medication-free and had no history of neurological or psychiatric disorders and drug/alcohol abuse. They were also not taking any medication or cigarette. We excluded the subjects with cardiovascular diseases that caused autonomic dysfunction, chronic diseases such as cancer or diabetes mellitus or hyperlipidemia. The subjects were told to refrain from brushing their teeth or eating for at least $60 \mathrm{~min}$ before the measurement session [8]. Using the previously developed method, one-time saliva sampling collection was conducted during the same time interval (2:00-3:00 PM) to minimize any effects of circadian rhythms.

We used a hand-held monitor (Nipro Co., Japan) and reagent paper to measure enzymatic sAA activity. This measurement method can be performed easily and quickly and is a convenient and useful objective indicator for medical and educational practice. The hand-held monitor consisted of a disposable test strip and a monitor $[11,12]$. This method for analyzing sAA has previously been evaluated and used $[13,14]$.

To assess autonomic functional changes, resting heart rate (HR) was measured in all subjects during the same time interval whilst they were lying in a recumbent position, as heart rate is thought to be an indicator of autonomic (sympathetic and parasympathetic) function.

The test consisted of three periods in which the subjects remained in a recumbent position: 10 min resting time (pre-test), 30 min fixed position time (test), and 10 min resting time (post-test). The subjects rested in a recumbent position on the floor for $10 \mathrm{~min}$ before the main part of the test. Then, they were required to not move or speak, as is seen in patients suffering from total paralysis, for $30 \mathrm{~min}$. They remained in the fixed position, alert, and quiet for $30 \mathrm{~min}$. Then, they were ordered to remain lying down and rest for a further $10 \mathrm{~min}$.

In addition, all subjects were asked whether they were experiencing any body pain or mental distress. Their pain and mental distress were estimated using a VAS (visual analogue scale). The VAS score was obtained from subjects who felt body pain in some body portion or mental distress at the end of the test. Subjects reported the level of pain and mental distress as VAS score.

Saliva samples were taken before the test, after the subjects had been lying in the fixed position for $30 \mathrm{~min}$, and at $10 \mathrm{~min}$ after the test. Heart rate was simultaneously measured in all subjects at the three time points.

The results are expressed as means $\pm \mathrm{SD}$. To compare the clinical variables at the three time points, the data were analyzed with repeated measures ANOVA by applying the SNK correction using Statview 5.01 (SAS Institute Inc., USA). The level of significance was set at $\mathrm{p}<0.05$.

\section{Results}

The volunteers' mean pre-test, test, and post-test sAA levels were $32.2 \pm 16.6(\mathrm{kU} / \mathrm{l})$ (range, 17-69), $61.5 \pm$ 47.2 (range, 24-193), and $34.9 \pm 11.8$ (range, 18-56), respectively. Significant differences were found using the ANOVA among three points $(\mathrm{F}=4.88, \mathrm{p}=0.018)$, therefore, SNK correction was conducted. There were significant differences between the test and pre-test sAA levels ( $\mathrm{p}<0.05$ ), and between the test and post-test sAA levels of $(p<0.05)$. The salivary alpha-amylase level increased significantly during the fixed positioning period and had returned to the baseline by $10 \mathrm{~min}$ after the test (Figure 1).

The mean pre-test, test, and post-test heart rates were $64.8 \pm 11.7,63.5 \pm 8.3$, and $61.2 \pm 10.4$, respectively. In contrast, heart rate did not change between the stress and the rest conditions (Table 1). Ten subjects (77\%) complained of body pain, particularly in the head, lumbar, hands, and heel. The mean VAS score of them for pain

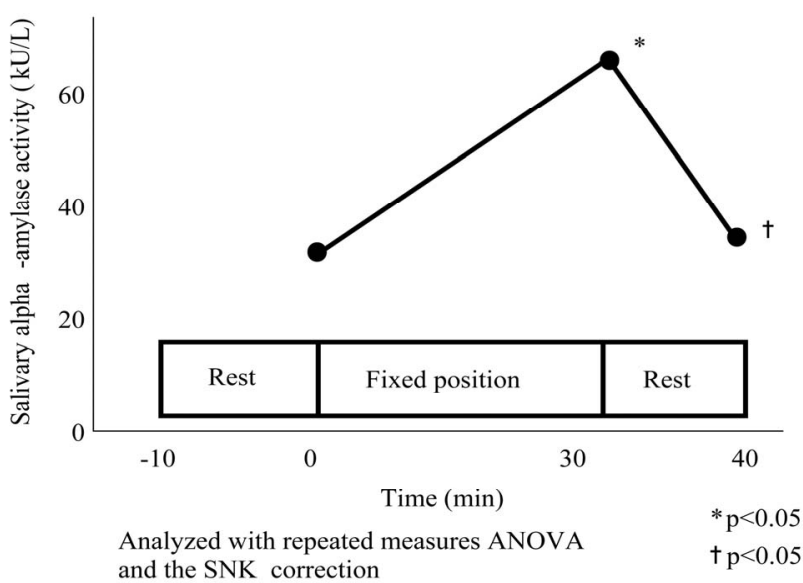

Figure 1. Changes in salivary alpha-amylase activity after remaining in a fixed position for $\mathbf{3 0}$ mins. 
Table 1. Psycho-physiological stress test results.

\begin{tabular}{cccc}
\hline & \multicolumn{3}{c}{ Time (min) } \\
\cline { 2 - 4 } & $\begin{array}{c}0 \\
\text { (Pre-test) }\end{array}$ & $\begin{array}{c}30 \\
\text { (Test) }\end{array}$ & $\begin{array}{c}40 \\
\text { (Post-test) }\end{array}$ \\
\hline $\begin{array}{c}\text { Salivary alpha-amylase } \\
(\mathrm{kU} / \mathrm{L})\end{array}$ & $32.2 \pm 16.6$ & $61.5 \pm 47.2^{*}$ & $34.9 \pm 11.8^{\dagger}$ \\
Heart rate & $64.8 \pm 11.7$ & $63.5 \pm 8.3$ & $61.2 \pm 10.4$ \\
\hline
\end{tabular}

${ }^{*} \mathrm{p}<0.05$; Pre-test v.s. Test $\quad \dagger \mathrm{p}<0.05$; Test v.s. Post-test

was 54. Mental distress was revealed in five subjects $(38 \%)$ and the mean VAS score of them for mental distress was 63 .

\section{Discussion}

In the present study, a marked increase in sAA activity due to physiological stress was observed during the main part of the test followed by a rapid return to the baseline level. Many subjects felt body pain and mental distress.

A portable sAA activity monitor has recently been developed, and several investigations have suggested that sAA reflects mental stress or have shown a positive relationship between the sAA increases and sympathetic activity.

Nater et al. [8] reported a positive relationship between the sAA level and sympathetic tone, which was assessed using heart rate variability parameters during periods of stress. Previous reports have also indicated that the sympathetic nervous system plays a predominant role in the sAA secretion observed under psychosocial stress $[2,3]$. Several reports have demonstrated that psychosocial stress increases the release of sAA and that a marked increase in sAA occurs following exposure to psychosocial stress, indicating that sAA activation is stress-dependent.

Shirasaki et al. [5] reported that sAA was significantly correlated with the VAS pain scale in patients with chronic pain. In addition, after pain therapy (epidural block), sAA activity was reduced in combination with a decrease in pain intensity but not SAM activity. Therefore, they suggested that sAA reflects the activity of the SAM system during pain-induced stress and that sAA measurements might be a good index for the objective assessment of pain intensity.

In psychiatric patients, we previously reported that the sAA activity of schizophrenic patients was significantly higher than that of control subjects, and the correlation between sAA level and psychiatric symptoms was highly significant [15].

These findings suggest that sAA correlates with psychiatric or/and psychosocial stress intensity.

To the best of our knowledge, only one study has proposed using biochemical measurements of sAA activity as a non-verbal communication method for interacting with children with severe motor and intellectual disabilities [13]. The physiological conditions of these children were evaluated by measuring their sAA activity, which required the daily insertion and removal of tracheostomy tubes or gastric tubes, and SAA was measured before and after medical care. As a result, they found that sAA activity was increased after medical care.

We performed the present study as a preliminary investigation aimed at clarifying whether this method could be used in clinical practice to evaluate the levels of psychosocial and/or physical stress in children with severe and intellectual disabilities, who are not able to move or complain about discomfort and/or distress. Such children can not move their bodies for themselves and may feel distress and pain when their body position is changed by assistance.

In this study, we examined the changes in the sAA levels of healthy volunteers subjected to conditions that were designed to reflect those experienced by children in the cerebral paralytic state, as it was impossible to directly examine the disabled children in fixed position for long time. There are no studies which examine the stress marker in the long-period fixed body position. We supposed that this particular situation could reflect the psychosocial and/or physical stress of the disabled children. The present results also suggest that the physiological conditions of patients who remain in a fixed position for a long period, who are considered to be in a similar condition to paralytic children/patients, can be evaluated by measuring their sAA levels.

On the basis of our results, we would like to use our method in clinical practice in future.

\section{Conclusion}

Salivary alpha-amylase is a useful parameter for evaluating psychophysiological stress in children with disabilities, since it is easy to measure. Thus, the changes in the sAA levels observed in the present study may reflect the level of mental stress experienced. Children with severe and intellectual disabilities can not fully express their emotions or communicate with their caregivers. This measurement method is useful for evaluating the stress levels of such patients.

\section{Acknowledgments}

The authors would like to thank the university students of Shimane University Faculty of Education for their support. 


\section{Conflicts of interest}

The authors state that there are no conflicts of interests that relate to this research.

\section{References}

[1] N. Takai, M. Yamaguchi, T. Aragaki, K Eto, K. Uchihashi and Y. Nishikawa, "Gender-specific Differences in Salivary Biomarker Responses to Acute Psychological Stress," Annals of the New York Academy of Sciences, Vol. 1098, 2007, pp. 510-515. doi:10.1196/annals. 1384.014

[2] E. B. Gordis, D. A. Granger, E. J. Susman and P. K. Trickett, "Asymmetry between Salivaly Cortisol and $\alpha$-maylase Reactivity to Stress: Relation to Aggressive Behavior in Adolescents," Psychoneuroendocrinology, Vol. 31, 2006, pp. 967-987. doi:10.1016/j.psyneuen.2006.05.010

[3] U. M. Nater and N. Rohlender, "Salivary Alpha-amylase as a Non-invasive Biomarker for the Sympathetic Nervous System: Current State of Research," Psychoneuroendocrinology, Vol. 34, 2009, pp. 486-496. doi:10.1016/j.psyneuen.2009.01.014

[4] N. Rohlender, U. M. Nater, J. M. Wolf, U. Ehlert and C. Kirshbaum, "Psychosocial Stress-induced Activation of Salivary Alpha-amylase. An Indicator of Sympathetic Activity?" Annals of the New York Academy of Sciences, Vol. 1032, 2004, pp. 258-263. doi:10.1196/annals.1314.033

[5] S. Shirasaki, H. Fujii, M. Takahashi, T. Sato, M. Ebina, Y. Noto and K. Hirota, "Correlation between SalivaryAmylase Activity and Pain Scale in Patients with Chronic Pain," Regional Anesthesia and Pain Medicine, Vol. 32, 2007, pp. 120-123.

[6] Y. Noto, T. Sato, M. Kudo, K. Kurata and K. Hirota, "The Relationship between Salivary Biomarkers and State Trait Anxiety Inventory Score under Mental Arithmetic Stress: A Pilot Study," Anesthesia \& Analgesia, Vol. 101, No. 6, 2005, pp. 1873-1876. doi:10.1213/01.ANE.0000184196.60838.8D

[7] U. M. Nater, N. Rohlender, J. Gaab, S. Berger, A. Jud, C. Kirshbaum and U. Ehlert, "Human Salivary Alpha-amylase Reactivity in a Psychosocial Paradigm," International Journal of Psychophysiology, Vol. 55, No. 3,
2005, pp. 333-342. doi:10.1016/j.ijpsycho.2004.09.009

[8] U. M. Nater, R. L. Marca, L. Florin, A. Moses, W. Langhans, M. M. Koller and U. Ehlert, "Stress-induced Changes in Human Salivary Alpha-amylase Activity-associations with Adrenergic Activity," Psychoneuroendocrinology, Vol. 31, 2006, pp. 49-58. doi:10.1016/j.psyneuen.2005.05.010

[9] A. van Stegeren, N. Rohleder, W. Everaerd and O. T. Wolf, "Salivary Alpha Amylase as Marker for Adrenergic Activity during Stress: Effect of Betablockade," Psychoneuroendocrinology, Vol. 31, 2006, pp. 137-141. doi:10.1016/j.psyneuen.2005.05.012

[10] C. Grillon, R. Duncko, M. F. Covington, L. Kopperman and M. A. Kling, "Acute Stress Potentiates Anxiety in Humans," Biological Psychiatry, Vol. 62, No. 10, 2007, pp. 1183-1186. doi:10.1016/j.biopsych.2007.06.007

[11] M. Yamaguchi, T. Kanemori, M. Kanemaru, N. Takai, Y. Mizuno and H. Yoshida, "Performance Evaluation of Salivary Amylase Activity Monitor," Biosensors and Bioelectronics, Vol. 20, No. 3, 2004, pp. 491-497. doi:10.1016/j.bios.2004.02.012

[12] M. Yamaguchi, M. Deguchi, J. Wakasugi, S. Ono, N. Takai, T. Higashi and Y. Mizuno, "Hand-held Monitor of Sympathetic Nervous System Using Salivary Amylase Activity and Its Validation by Driver Fatigue Assessment," Biosensors and Bioelectronics, Vol. 21, No. 7, 2006, pp. 1007-1014. doi:10.1016/j.bios.2005.03.014

[13] T. Higashi, Y. Mizuno, M. Oonishi, K. Takeda and M. Yamaguchi, "Nonverbal Communication Tool for Children with Severe Motor and Intellectual Disabilities Using Biochemical Measurement," Conference Proceedings - IEEE Engineering in Medicine and Biology Society, Vol. 4, 2005, pp. 3538-3541.

[14] M. Yamaguchi, J. Wakasugi and J. Sakakima, "Competitive and Product Inhibition-based $\alpha$-amylase Activity Analysis Method," Clinical Biochemistry, Vol. 41, No. 4-5, 2008, pp. 325-330. doi:10.1016/j.clinbiochem.2007.10.016

[15] T. Inagaki, T. Miyaoka, S. Okazaki, H. Yasuda, T. kawamukai, E. Utani, R. Wake, M. Hayashida, J. Horiguchi and S. Tsuji, "High Salivary Alpha-amylase Levels in Patients with Schizophrenia: A Pilot Study," Progress in Neuro-Psychopharamacology \& Biological Psychiatry, Vol. 34, No. 4, 2010, pp. 688-691. doi:10.1016/j.pnpbp.2010.03.024 